\title{
Australian Survey of Current Practice and Guideline Use in Adult Cancer Pain Assessment and Management: Perspectives of Palliative Care Physicians
}

\author{
Melanie Lovell, MBBS, PhD, FRACP, FAChPM,1,6 Meera Agar, MBBS, MPallCare, FRACP, FAChPM,1,2 \\ Tim Luckett, BSc(Hons), PhD, ${ }^{3,4}$ Patricia M. Davidson, BA, Med, PhD, ${ }^{3,5}$ \\ Anna Green, BSocSci, MDev, ${ }^{3,5}$ and Josephine Clayton, MBBS, PhD, FRACP, FAChPM ${ }^{1,6}$
}

\begin{abstract}
Background: Cancer pain continues to be undertreated, despite the availability of evidence-based guidelines. The Australian National Pain Strategy identified establishment of systems and guidelines to adequately manage cancer pain as a high priority.

Objectives: This study aimed to identify barriers and facilitators to adult cancer pain assessment and management as perceived by Australian health professionals; establish the perceived need for new Australian guidelines and implementation strategies; identify which guidelines are used; and identify barriers and facilitators to guideline use. This article focuses on the perceptions of responding palliative care physicians.

Design: A cross-sectional survey was administered online.

Participants: Invitations were circulated via peak bodies and clinical leaders. Comments were coded independently by two researchers.

Results: Ninety-two palliative care physicians responded to the survey; $39 \%$ of the national total. The majority reported barriers to pain management, including insufficient access to nonpharmacologic interventions, poor coordination between services, and management challenges posed by comorbidities. Forty-five percent reported using pain guidelines, most commonly the Australian Therapeutic Guidelines-Palliative Care. Respondents were largely supportive of the development of new Australian guidelines and implementation strategies, in particular any offering advice on specific cases of cancer pain (e.g., neuropathic), patient self-management resources, assessment of patient priorities, and disciplinary roles.

Conclusion: Barriers to evidence-based practice identified by our survey might be addressed via strategies to support decision making and coordination of care (e.g., a clinical pathway). Particular attention should be paid to promoting access to nonpharmacologic interventions and patient education, and improving referral and care coordination.
\end{abstract}

\section{Introduction}

$\mathbf{P}$ AIN IS EXPERIENCED by up to $75 \%$ of people with cancer yet remains undertreated across the world. ${ }^{1-5}$ Failure to manage pain is due to barriers at all levels-patient, caregiver, health professional, and health care system. ${ }^{6-17}$ Research suggests that implementation of evidence-based clinical practice guidelines for cancer pain can improve the processes of care and patient outcomes. ${ }^{11}$ A systematic review has identified three models that have demonstrated efficacy to some degree: (1) institutional models, which provide policies and procedures for regular pain assessment and standardization of pain treatment; (2) clinical pathways, which provide step-by-step guidance on optimal sequencing and timing of assessment and management; and (3) expert consultation. ${ }^{11}$

\footnotetext{
${ }^{1}$ Department of Palliative Care, HammondCare, Sydney, New South Wales, Australia.

${ }^{2}$ Department of Palliative and Supportive Services, Daw Park, Adelaide, South Australia, Australia.

${ }^{3}$ Centre for Cardiovascular and Chronic Care, ${ }^{4}$ Faculty of Nursing and Midwifery, ${ }^{5}$ Faculty of Health, University of Technology, Sydney, New South Wales, Australia.

${ }^{6}$ Department of Palliative and Supportive Care, Greenwich Hospital, University of Sydney, Sydney, New South Wales, Australia.

Accepted June 21, 2013.
} 
In Australia, pain in people with cancer has been identified as an important area for improvement by both the National Institute of Clinical Studies (NICS) and the Cancer Institute New South Wales (NSW). ${ }^{18,19}$ The recently launched Australian National Pain Strategy has six major goals including, "timely access to best-practice, evidence-based assessment and care." ${ }^{20}$ The Pain Strategy was developed in 2010 at a National Pain Summit and included input from a Cancer Pain and Palliative Care Working Group. This group concluded that a primary objective should be the promotion of guidelines and systems to ensure adequate assessment and management of cancer pain, improve communication between patients and health care providers, and facilitate coordination between providers. However, more data are needed to guide selection of appropriate strategies. To date, Australian studies examining barriers to cancer pain assessment and management have focused on the perceptions of patients and caregivers. ${ }^{21-24}$ These surveys identified patient and caregiver barriers in the form of misconceptions about opioids, perceived lack of control, poor management expertise, and barriers to communication. A better understanding of barriers and facilitators from the perspectives of Australian health professionals is needed to inform promotion of evidencebased practice.

The aims of the current study were to:

1. Inform understanding of barriers and facilitators to adult cancer pain assessment and management in various Australian clinical practice settings from the perspectives of different disciplines.

2. Establish the level of support for new Australian guidelines and associated implementation strategy.

3. Find out which guidelines for adult cancer pain are used.

4. Inform understanding of barriers and facilitators to adult cancer pain guideline use.

We were especially interested in the views of motivated clinicians most likely to drive change. ${ }^{25}$

\section{Methods}

The methods have been described in detail elsewhere. ${ }^{26}$ Briefly, the study used a cross-sectional online survey design. Participants were health professionals of any discipline involved in caring for adults with cancer pain in Australia. This article focuses on responses from participants who selfreported their discipline as palliative care physician. Palliative care physicians are generally considered experts in pain management and provide clinical care for people with advanced cancer who are experiencing symptoms, especially as they approach the end of life. Understanding their current practice and attitudes is therefore critical for any efforts aimed at improving pain assessment and management.

Participants were recruited via e-mail invitations and newsletters sent out by peak bodies and other organizations (see Table 1 for those that circulated to palliative care physicians). We also asked Australian clinical leaders in cancer pain to circulate the invitation via their networks.

An open online survey was administered via a secure online platform, SurveyMonkey ${ }^{\circledR}$ (www.surveymonkey.com/). Open online surveys are subject to selection bias because participants self-select, leading to a volunteer effect. ${ }^{27}$ In the
Table 1. Peak Bodies that Circulated Survey to Palliative Care Physicians

ANZ Society of Palliative Medicine (ANZSPM)

Australasian Chapter of Palliative Medicine

Australian Pain Society

CanNET NT

CareSearch

Clinical Oncological Society of Australia

Grampian Integrated Cancer Service

Painaustralia

Palliative Care Australia

Primary Care Collaborative Cancer Clinical Trials Group

(PC4)

WA Centre for Cancer \& Palliative Care

current study, an overrepresentation of motivated respondents with experience of, and interest in, guideline use were considered to be supportive of, rather than disabling to, our aims.

The survey "went live" on August 30, 2011 and closed on April 30, 2012. Information about respondents requested in the survey included discipline and primary workplace location (state/territory, postcode), sector, and outreach status. Respondents were asked how routinely their primary workplace implemented each of a list of evidence-based practices identified by Dy et al. ${ }^{28}$ and the Clinical Indicators for Pain Project funded by the Victorian Department of Health. ${ }^{29}$ Further questions related to access to specialist palliative care and pain services; use of and familiarity with guidelines for assessment and management of cancer pain; the need for new Australian guidelines and implementation strategies; and perceptions of barriers and facilitators to best practice and use of guidelines.

Item response options included yes/no, verbal rating scales (e.g., strongly agree, agree, disagree, strongly disagree) and comment boxes after every item. Comments were independently coded by two authors (M.L. and T.L.) who then met to reach consensus.

\section{Results}

Ninety-two palliative care physicians were recruited to the survey. This represents 39\% of the total 236 palliative medicine specialists registered with the Australian Health Practitioner Regulation Agency. ${ }^{30}$ Ten participants self-identified as holding dual specialty roles as oncologists, four as registrars / trainees, three as geriatricians, two as general practitioners, two as pain specialists, and one as registered nurse. The geographic spread of respondents roughly corresponded to that of the general population, ${ }^{31}$ with all states and territories represented except the Northern Territory (Table 2). Thirtyfive percent of respondents provided an outreach clinic to a regional or remote area from a metropolitan hospital. Only 7\% were in private practice.

Palliative care physicians self-report close adherence to current recommendations in cancer pain assessment and management, despite many barriers identified. The most prevalent of these appears to relate to lack of access to services to provide nonpharmacologic management of cancer pain. These include: psychologists providing cognitive behavioral therapy (CBT) for which there exists Level 1 evidence for 
Table 2. Sample Characteristics

\begin{tabular}{lr}
\hline $\begin{array}{l}\text { State/territory }(\% \text { Australian } \\
\left.\text { general population }{ }^{\mathrm{a}}\right)\end{array}$ & $(\mathrm{n}=92) \%$ \\
\hline VIC (24.9) & $28 \%$ \\
NSW (32.3) & $27 \%$ \\
QLD (20.2) & $20 \%$ \\
WA (10.4) & $8 \%$ \\
SA (7.3) & $3 \%$ \\
TAS (2.3) & $7 \%$ \\
ACT (1.6) & $2 \%$ \\
NT (1.0) & $0 \%$ \\
Sector & \\
Public hospital & $43 \%$ \\
Public practice & $40 \%$ \\
Private practice & $4 \%$ \\
Private hospital & $3 \%$ \\
Community care & $3 \%$ \\
Does the physician run an outreach clinic & \\
Yes & $35 \%$ \\
\hline
\end{tabular}

${ }^{\text {a}}$ Based on demographic data from the Australian Bureau of Statistics. ${ }^{1}$ efficacy in cancer pain management; and physiotherapists who provide therapy for musculoskeletal pain. Many of the free text comments described health professionals' lack of knowledge about opioids and their use. The next most prevalent barrier identified was a lack of coordination across multiple providers.

Availability of more patient and caregiver education was most frequently nominated as being likely to improve cancer pain assessment and management at the physician's primary workplace (Table 3).

Lack of available specialist pain services and long waiting times for these services were identified as causing delayed referral to those services by $62 \%$ of 29 responding palliative care physicians. Access to a pain service was of acceptable speed and ease for only $44 \%$ of 81 respondents (Table 4 ).

Only $68 \%$ of palliative care physicians felt that all or nearly all people with cancer pain should be seen by palliative care services indicating that $32 \%$ felt that palliative care services should be used for only a select group of patients who could not be managed adequately by oncologists or general practitioners. Only eight respondents answered the question about delayed referrals to palliative care services possible because

Table 3. Responses to Questions on Current Practice in Assessment and Management of Cancer Pain

How routinely does each of these practices takes place at your primary workplace?

$(n=77) \%$

(Most of the time/always or nearly always)

Use of breakthrough opioids in cancer patients receiving long-acting opioids

Use of bowel regimens in cancer patients receiving opioids

Follow-up of pain management for cancer patients

Scheduled pain medication for severe pain

Continuity of opioid doses across health care settings

Regular pain assessment in cancer patients

Routine assessment of pain in new cancer patients

Routine pain education for cancer patients

Use of a validated pain scale to assess cancer pain

$99 \%$

$96 \%$

$97 \%$

$95 \%$

$92 \%$

$91 \%$

$91 \%$

$83 \%$

$71 \%$

$(n=76) \%$

of a barrier/very substantial barrier)

Difficulty accessing services that enable nonpharmacologic management of cancer pain

Lack of coordination across multiple providers

Challenges posed by comorbidities

Difficulty accessing interventional pain services

Impact of distance on ability to access pain-related services for patients

Inability to access ongoing data on pain and quality of life to monitor progress and outcomes

Lack of regular case reviews to critically discuss and evaluate pain assessment and management

Difficulty keeping abreast of advances from research

Inadequate remuneration for time spent (e.g., on multidisciplinary meetings)

Lack of benchmarks to assess improvement in quality of pain management over time

$83 \%$

$78 \%$

$76 \%$

$71 \%$

$62 \%$

$62 \%$

$58 \%$

$58 \%$

$54 \%$

$54 \%$

$47 \%$

$47 \%$

$30 \%$

Difficulty accessing acute pain services

Limited expertise in the assessment and management of cancer pain

Difficulty accessing palliative care services

What would improve cancer pain assessment and management at your primary workplace? (Check as many as apply)

More patient education

More caregiver education

More training and access to information on pain assessment and management

Increase in dedicated clinician time

One or more clinical champion(s)

A more multidisciplinary approach

New mechanisms for evaluating outcomes

Policy changes

Unsure
$11 \%$

$(n=77) \%$

$64 \%$

$62 \%$

$57 \%$

$55 \%$

$42 \%$

$39 \%$

$18 \%$

$10 \%$

$5 \%$ 
Table 4. Responses to Questions Regarding Specialist Pain Service Involvement in Cancer Pain Assessment and Management

\begin{tabular}{lr}
\hline Question (Response) & $(\mathrm{n}=77) \%$ \\
\hline What proportion of patients with cancer pain would benefit from referral to a specialist pain & \\
service for assessment and management? (Most/all or nearly all) & $\begin{array}{l}\text { (n) } \\
\text { In your area health service, how quickly and easily can a specialist pain service typically be }\end{array}$ \\
accessed for assessment and management of cancer pain? (Access is of acceptable speed and ease) & $44 \%$ \\
How often is referral to a specialist pain service for assessment and management of cancer & $(n=29) \%$ \\
pain delayed for the following reasons? (Most of the time/always or nearly always) & $62 \%$ \\
Lack of available specialist services & $62 \%$ \\
Long waiting time & $31 \%$ \\
Need for patients to travel to tertiary centers & $24 \%$ \\
Difficult/complex referral processes & $21 \%$ \\
Lack of coordination between services & $17 \%$ \\
Difficulty accessing patient transport & $14 \%$ \\
Lack of knowledge of available services & $7 \%$ \\
Difficulty selecting which patients should be referred & $3 \%$ \\
Delays are due to patients themselves (e.g., because they fear perceived side effects & \\
or addictiveness of treatment) &
\end{tabular}

the options given in the questions were not barriers for other respondents (Table 5).

\section{Guideline use}

More than $90 \%$ of 77 responding palliative care physicians supported the need for guidelines and an implementation strategy for those guidelines. A guideline was already in routine use in the practice of $45 \%$ of 87 respondents. The (Australian) Therapeutic Guidelines-Palliative Care was the most commonly used guideline used by 22 respondents (Table 6).

\section{Discussion}

This was the first study to explore Australian palliative care physicians' perceptions of ways to improve cancer pain assessment and management. Palliative care physicians responding to our online survey were strongly supportive of the need for new Australian cancer pain assessment and man- agement guidelines and an implementation strategy. When asked about barriers to provision of pain management, respondents identified a lack of access to nonpharmacologic pain management strategies, lack of coordination between providers, and patient comorbidities. Other barriers included lack of consensus and knowledge about pain management and lack of resources to teach.

The most commonly identified barrier, lack of access to nonpharmacologic pain management strategies, has also been identified as a barrier in other countries. ${ }^{32}$ Nonpharmacologic pain management strategies are recommended briefly in many international guidelines ${ }^{33,34}$ and could be included in more detail in an Australian guideline. Inclusion of such strategies in a national guideline may influence providers and administrators to increase access to services enabling nonpharmacologic pain management, including physiotherapy, occupational therapy, and psychology. Psychology, in particular, while increasingly available in oncology departments, remains uncommon in Australian specialist palliative care

Table 5. Responses to Questions Regarding Specialist Palliative Care Service Involvement in Cancer Pain Assessment and Management

Question (Response)

$(\mathrm{n}=76) \%$

What proportion of patients with cancer pain would benefit from referral to a specialist palliative $68 \%$ care service for assessment and management? (Most/all or nearly all)

In your area health service, how quickly and easily can a specialist palliative care service typically be accessed for assessment and management of cancer pain? (Access is of acceptable speed and ease)

How often is referral to a specialist palliative care service for assessment and management of cancer pain delayed for the following reasons? (Most of the time/always or nearly always)

Lack of knowledge of available services

Difficulty selecting which patients should be referred

Lack of coordination between services

Delays are due to patients themselves (e.g., because they fear perceived side effects or addictiveness of treatment)

Long waiting time

Difficult/complex referral processes

Difficulty accessing patient transport

Need for patients to travel to tertiary centers

$(n=75) \%$

$87 \%$

$(n=8) \%$

$13 \%$

$13 \%$

$13 \%$

$0 \%$

$0 \%$

$0 \%$

$0 \%$

$0 \%$ 
Table 6. Responses to Questions on Guidelines for Assessing and Managing Cancer Pain

Question (Response)

What level of need do you think there is for the following? (Some need/urgent need)

An Australian guideline for nonpharmacologic management of cancer pain

$(\mathrm{n}=77) \%$

$92 \%$

An Australian guideline for pharmacologic management of cancer pain

$90 \%$

Implementation strategy to inform use of existing guidelines in and across different service settings

$90 \%$

An Australian guideline for assessing cancer pain

$90 \%$

In a new Australian implementation strategy for guidelines on cancer pain, how useful would

the following be? (Somewhat/very useful)

Advice on best practice for specific cases of cancer pain (e.g., nociceptive)

Inclusion of patient 'action plans' to aid self-management

Guidance on assessing patient-identified priorities

Guidance on roles for each discipline

Templates for taking case histories and identifying patient-identified priorities

Patient version of implementation strategy

Inclusion of key performance indicators

$(n=77) \%$

One or more guideline routinely used in primary workplace?

Yes

Which specific guideline(s) used routinely in primary workplace

(Australian) Palliative Care Expert Group (2010): Therapeutic Guidelines— Palliative Care. Version 3

Institutional or departmental guideline(s)

NHMRC (2006): Guidelines for a palliative approach in residential aged care

European Association for Palliative Care (2001): Morphine and alternative opioids in cancer pain; the EAPC recommendations

American Pain Society (2005): Guidelines for the management of cancer pain in adults and children

European Society for Medical Oncology (2007): Minimum clinical recommendations for the management of cancer pain

National Comprehensive Cancer Network (2010): Clinical practice guidelines in Oncology - adult cancer pain

National Institutes of Health (2002): Symptom management in cancer: pain, depression and fatigue

Palliative Care in Paediatric Oncology

APSOC Guidelines for the management of neuropathic pain (APSOC-Management in pain in residential aged care facilities)

Therapeutic guidelines

Palliative Care Formulary 3rd Edition Twycross \& Wilcock

$96 \%$

$95 \%$

$92 \%$

$90 \%$

$84 \%$

$79 \%$

$74 \%$

$(n=87) \%$

$45 \%$

$(n=32) \%$

$66 \%$

$31 \%$

$16 \%$

$13 \%$

$9 \%$

$3 \%$

$3 \%$

$3 \%$

$3 \%$

$3 \%$

Twycross Therapeutics in Terminal Cancer

Cancer Institute EVIQ

Internal guidelines and policies are used regarding analgesia and analgesics

Burst Ketamine Guidelines Methadone Guidelines (Perth)

Paediatric Palliative Care references

Own silver chain guidelines, developed from looking at other published info

Peter Mac Cancer Pain Management Guidelines

Peter MacCallum Guidelines Western Health Victoria Guidelines

Victorian Eastern Metropolitan Regional Palliative Care Consortia Opioid Conversion Guidelines

We have developed our own guidelines for the management of uncontrolled cancer pain

NHMRC Acute Pain Guidelines

Analgesics Therapeutic Guidelines-Palliative Care

Perceptions about guideline use (Agree/strongly agree)—completed by

One or more guideline working well

There are adequate resources at my primary workplace to provide care according $78 \%$

to one or more pain guideline(s)

My service endorses use of one or more particular pain guideline(s)

$78 \%$

Clinical staff at my primary workplace adhere to the same pain guideline(s)

$72 \%$

services. A problem with access to medicines, in particular items not subsidized by the Pharmaceutical Benefits Scheme such as gabapentin for pain management was also identified by respondents.

The second most commonly identified barrier, lack of coordination across multiple providers, confirms the priority placed on the need for improvement in this area by the National Pain Summit. ${ }^{18}$ Respondents reported that poor coordination resulted in medications being omitted either by accident or intentionally by providers who are reluctant to prescribe opioids. Increased collaboration between specialties through joint clinics, ward rounds and dual training of registrars between palliative care and pain medicine or palliative care and oncology may help to improve care coordination.

A guideline and implementation strategy can take the form of a clinical pathway. On the basis of the perceived need and identified evidence-practice gap, the authors are currently 
developing such a clinical pathway based on international guidelines and a consensus process involving Australian experts in cancer pain management (http://wiki.cancer.org. au/australia/Guidelines:Cancer_pain_management). It will include patient screening with self-report measures, health professional education, and a patient-held version of the pathway. It will be patient-centered, evidence-based and updated, and include opioid conversion information. It will act as an education tool about opioids for consumers and health professionals and address many of the commonly held misconceptions about opioids.

Further strategies used by the clinical pathway for cancer pain will include recommendations for a link between pain assessment and evidence-based management being incorporated into hospital accreditation, regular prompts to use the guidelines, and measures designed to foster good organizational support. While concern has been expressed ${ }^{35,36}$ that clinical guidelines create a barrier to critical thinking, the main aim of guidelines and pathways is to ensure that essential elements of comprehensive pain assessment and management are not omitted by those who lack specialist knowledge.

Limitations to the survey reported here include the fact that only 39\% of Australian palliative care physicians responded and some questions were answered by a small proportion of respondents only as seen in the tables. Participants may not have answered questions pertaining to areas which were not seen as important or relevant in their practice but as the reasons are not given, it is difficult to draw conclusions for individual items with a low response rate. The methods used to recruit participants and administer the survey are likely to have resulted in a volunteer effect. The results are likely to reflect the views of "clinical champions" who would support implementation of strategies for improving pain assessment and management, but it would also be informative to collect data on the opinions of less proactive physicians. There are no data to support the high reported adherence to evidencebased practice. There is evidence that pain is undertreated across a range of settings, which would suggest that there is scope for practice improvement.

\section{Conclusion}

Australian palliative care physicians support an implementation strategy for cancer pain management guidelines. An example of a guideline and implementation strategy is a clinical pathway which can standardize pain management recommendations and be a valuable tool for health professional education in a wide variety of settings and for improving communication about pain assessment and management. Increased awareness of the gaps in availability of evidence-based strategies may also influence policy makers.

\section{Acknowledgments}

We would like to acknowledge staff and postgraduate students at the Faculty of Health at UTS for comments on draft versions of the survey reported in this article. We would also like to acknowledge Alex Brando from the Peter MacCallum Cancer Centre for sharing information about the Clinical Indicators for Pain Project funded by the Victorian Department of Health. Finally, we would like to thank the peak bodies and clinical experts referred to in the Methods section for assisting with recruitment to the survey.

\section{Author Disclosure Statement}

Melanie Lovell has received a peer-reviewed Cancer Research Grant from Pfizer.

\section{References}

1. van den Beuken-van Everdingen MH, de Rijke JM, Kessels AG, Schouten HC, van Kleef M, Patijn J: Prevalence of pain in patients with cancer: A systematic review of the past 40 years. Ann Oncol 2007;18:1437-1449.

2. Foley KM: How well is cancer pain treated? Palliat Med 2011;25:398-401.

3. Deandrea S, Montanari M, Moja L, Apolone G: Prevalence of undertreatment in cancer pain. A review of published literature. Ann Oncol 2008;19:1985-1991.

4. Stockler MR, Wilcken NRC: Why is management of cancer pain still a problem? J Clin Oncol 2012;30:1907-1908.

5. Fisch MJ, Lee JW, Weiss M, Wagner LI, Chang VT, Cella D, Manola JB, Minasian LM, McCaskill-Stevens W, Mendoza TR, Cleeland CS. Prospective, observational study of pain and analgesic prescribing in medical oncology outpatients with breast, colorectal, lung, or prostate cancer. J Clin Oncol 2012;30:1980-1988.

6. Herr K, Titler M, Fine P, Sanders S, Cavanaugh J, Swegle J, Forcucci $\mathrm{C}$, Tang X. Assessing and treating pain in hospices: Current state of evidence-based practices. J Pain Symptom Manage 2010;39:803-819.

7. Cohen MZ, Easley MK, Ellis C, Hughes B, Ownby K, Rashad BG, Rude M, Taft E, Westbrooks JB; JCAHO: Cancer pain management and the JCAHO's Pain Standards: An institutional challenge. J Pain Symptom Manage 2003;25:519-527.

8. Fazeny B, Muhm M, Hauser I, Wenzel C, Mares P, Berzlanovich A, Hagmeister $\mathrm{H}$, Marosi C: Barriers in cancer pain management. Wien Klin Wochenschr 2000;112:978-981.

9. Jacobsen R, Sjøgren P, Møldrup C, Christrup L: Physicianrelated barriers to cancer pain management with opioid analgesics: a systematic review. J Opioid Manag 2007;3:207-214.

10. Pargeon KL, Hailey BJ. Barriers to effective cancer pain management: A review of the literature. J Pain Symptom Manage 1999;18:358-368.

11. Brink-Huis A, van Achterberg T, Schoonhoven L: Pain management: A review of organisation models with integrated processes for the management of pain in adult cancer patients. J Clin Nurs 2008;17:1986-2000.

12. Sun VC, Borneman T, Ferrell B, Piper B, Koczywas M, Choi $\mathrm{K}$ : Overcoming barriers to cancer pain management: an institutional change model. J Pain Symptom Manage 2007;34:359-369.

13. Jacobsen R, Møldrup C, Christrup L, Sjøgren P. Patientrelated barriers to cancer pain management: A systematic exploratory review. Scand J Caring Sci 2009;23:190-208.

14. Meeker MA, Finnell D, Othman AK: Family caregivers and cancer pain management: A review. J Fam Nurs 2011;17:29-60.

15. Oldenmenger WH, Sillevis Smitt PA, van Dooren S, Stoter G, van der Rijt CC: A systematic review on barriers hindering adequate cancer pain management and interventions to reduce them: A critical appraisal. Eur J Cancer 2009;45:13701380.

16. Sun V, Borneman T, Piper B, Koczywas M, Ferrell B: Barriers to pain assessment and management in cancer survivorship. J Cancer Survivor 2008;2:65-71.

17. Breuer B, Fleishman SB, Cruciani RA, Portenoy RK: Medical oncologists' attitudes and practice in cancer pain management: A national survey. J Clin Oncol 2011;29:4769-4775. 
18. National Institute of Clinical Studies: Evidence-Practice Gaps Report Volume 1: A Review of Developments 2004-2007. Canberra: National Health and Medical Research Council, 2008.

19. Heading G, et al. New South Wales Cancer Patient Satisfaction Survey. Interim Report. Sydney: Cancer Institute, 2008.

20. National Pain Summit Initiative: National Pain Strategy. Melbourne: Faculty of Pain Medicine, 2010.

21. Aranda S, Yates P, Edwards H, Nash R, Skerman H, McCarthy A: Barriers to effective cancer pain management: A survey of Australian family caregivers. Eur J Cancer Care (Engl) 2004;13):336-343.

22. Yates $P$, Aranda $S$, Edwards $H$, Nash $R$, Skerman $H$, McCarthy A: Family caregivers' experiences and involvement with cancer pain management. J Palliat Care 2004;20:287-296.

23. Yates PM, Edwards HE, Nash RE, Walsh AM, Fentiman BJ, Skerman HM, Najman JM: Barriers to effective cancer pain management: A survey of hospitalized cancer patients in Australia. J Pain Symptom Manage 2002;23:393-405.

24. Potter VT, Wiseman CE, Dunn SM, Boyle FM: Patient barriers to optimal cancer pain control. Psychooncology 2003;12:153-150.

25. National Institute for Health and Clinical Excellence: Behaviour Change at Population, Community and Individual Levels. NICE Public Health Guidance. London: NICE, 2007.

26. Luckett T, Davidson PM, Boyle F, Liauw W, Agar M, Green A, Lovell M; ImPaCCT (Improving Palliative Care through Clinical Trials) and PoCoG (Psycho-oncology Co-operative Research Group) Symptom Cluster Collaborative: Australian survey of current practice and guideline use in adult cancer pain assessment and management: Perspectives of oncologists. Asia Pac J Clin Oncol (in press).

27. Eysenbach G, Wyatt J: Using the Internet for surveys and health research. J Med Internet Res 2002;4:E13.

28. Dy SM, Asch SM, Naeim A, Sanati H, Walling A, Lorenz KA: Evidence-based recommendations for cancer pain management. J Clin Oncol 2008;26:3879-3785.
29. Brando A (ed): Transforming literature findings to practical indicators-An effort to incorporate best practice pain management for palliative care patients [abstract 418]. Annual Scientific Meeting of the Clinical Oncological Society of Australia (COSA); 2011; Perth: Asia-Pacific Journal of Clinical Oncology.

30. Australian Health Practitioner Regulation Agency. AHPRA web enquiry. 2012.

31. Australian Bureau of Statistics. Australian Demographic Statistics (3101.0). Canberra: ABS2011.

32. Luckett T, Davidson PM, Green A, Boyle F, Stubbs J, Lovell M:. Assessment and management of adult cancer pain: A systematic review and synthesis of recent qualitative studies aimed at developing insights for managing barriers and optimizing facilitators within a comprehensive framework of patient care. J Pain Symptom Manage 2013;46:229-253.

33. Scottish Intercollegiate Network (SIGN): Control of Pain in Patients with Cancer: A National Clinical Guideline. Report No. 106. Edinburgh: 2008.

34. National Comprehensive Cancer Network (NCCN): NCCN Clinical Practice Guidelines in Oncology: Adult Cancer Pain. Version 2.2011. New York: NCCN2011.

35. Nanan RK, Poulton AS, Champion BL: Clinical pathways: A departure from the art of medicine. Med J Aust 2012;196:96.

36. Dietz HP, Stokes BJ: A plea for professional independence. Med J Aust 2012;196:104-105.

Addess correspondence to: Melanie Lovell, MBBS, PhD, FRACP, FAChPM PO Box 5084 Greenwich, New South Wales 2065 Australia

E-mail: Mlovell@hammond.com.au 